\title{
Revista Española de
}

\section{Nutrición Humana y Dietética}

\section{Spanish Journal of Human Nutrition and Dietetics}

www.renhyd.org

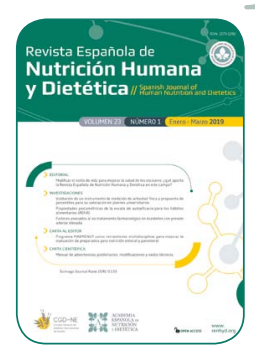

\section{ARTÍCULO DE INVESTIGACIÓN}

\section{Propiedades psicométricas de la escala de autoeficacia para los hábitos alimentarios (AEHA)}

\author{
Jaqueline Garcia da Silva ${ }^{a, *}$, María Isabel Peralta-Ramírez ${ }^{a}$, Nuria Navarrete-Navarrete ${ }^{b}$, \\ Daniele Silva-Silva ${ }^{c}$, Guilherme Welter Wendt ${ }^{d}$, Vicente E Caballo ${ }^{a}$ \\ a Departamento de Personalidad, Evaluación y Tratamiento Psicológico, Facultad de Psicología, Universidad de Granada, \\ Granada, España. \\ b Unidad de Gestión Clínica de Medicina Interna, Complejo Hospitalario Universitario de Granada, Granada, España. \\ Faculdade de Psicologia, Pontifícia Universidade Católica do Rio Grande do Sul, Porto Alegre, Brasil. \\ d Unit for School and Family Studies, Department of Psychology, Goldsmiths, University of London, Londres, Reino Unido. \\ *garcia.jake@hotmail.com
}

Editora Asignada: Véronica Dávila-Batista. Universidad de León. León, España.

Recibido el 16 de julio de 2018; aceptado el 12 de febrero de 2019; publicado el 22 de febrero de 2019.

\section{PALABRAS CLAVE}

Autoeficacia;

Conducta

Alimentaria;

Síndrome

Metabólico;

Psicometría

Propiedades psicométricas de la escala de autoeficacia para los hábitos alimentarios (AEHA)

\section{RESUMEN}

Introducción: El objetivo de este trabajo fue describir las propiedades psicométricas preliminares de la "Escala de autoeficacia para hábitos alimentarios".

Material y Métodos: Participaron en un ensayo clínico aleatorizado para la modificación del estilo de vida 135 sujetos con síndrome metabólico, divididos en dos grupos (grupo control y grupo experimental), con un seguimiento de 18 meses. La evaluación incluyó datos antropométricos, clínicos, psicológicos y de estilo de vida. Para la validez y fiabilidad se realizó análisis factorial, de la consistencia interna, estabilidad temporal, sensibilidad al cambio y validez concurrente.

Resultados: La solución de 2 factores explica el 60,97\% de la varianza común. La consistencia interna para la puntuación total fue de 0,971 - alfa de Cronbach. En cuanto a la estabilidad temporal, no se observó ningún cambio en el grupo control. Con respecto a la sensibilidad al cambio, el grupo experimental presentó mayor autoeficacia a lo largo del seguimiento.

Conclusiones: Los resultados preliminares mostraron niveles adecuados de validez y fiabilidad, demostrando ser una escala útil para evaluar la autoeficacia relacionada a la alimentación. 


\section{Psychometric properties of Self-Efficacy to Regulate Eating Habits (SEREH) scale}

\section{KEYWORDS}

Self Efficacy;

Feeding Behavior;

Metabolic

Syndrome;

Psychometrics.

\section{ABSTRACT}

Introduction: The aim of this study was to describe the preliminary psychometric properties of the "Self-Efficacy to Regulate Eating Habits (SEREH)".

Material and Methods: Participated in a randomized clinical trial for lifestyle modification 135 subjects with metabolic syndrome, who were divided into two groups (e.g., experimental and control), in a 18-months follow-up. The evaluation included anthropometric, clinical, psychological and lifestyle data. To verify the validity and reliability, a factorial analysis was performed, as well as analyses of the internal consistency, temporal stability, sensitivity to change, and convergent validity.

Results: A solution with two factors was extracted, which explained $60.97 \%$ of the variance of the SEREH. The internal consistency for the total score was 0,971 - Cronbach's alpha. Regarding temporal stability, no changes were observed during the follow-up in the control group. With regard to sensitivity to change, the experimental group showed greater self-efficacy than did the control group during the follow-up.

Conclusions: These preliminary results showed adequate psychometric properties of the scale, demonstrating that this is a valid and reliable scale for assessing self-efficacy related to food behaviors.

\section{CITA}

Garcia da Silva J, Peralta-Ramírez MI, Navarrete-Navarrete N, Silva-Silva D, Welter Wendt G, Caballo VE. Propiedades psicométricas de la escala de autoeficacia para los hábitos alimentarios (AEHA). Rev Esp Nutr Hum Diet. 2019; 23(1): 14-26. doi: 10.14306/renhyd.23.1.627

\section{INTRODUCCIÓN}

La prevalencia del síndrome metabólico (SM) en EE.UU. oscila entre el 20 y el $30 \%$ y en España las cifras se mantienen aproximadas. La intervención terapéutica de primera línea para el SM es la modificación del estilo de vida (reducción del peso, alimentación saludable y actividad física regular $)^{1,2}$. La autoeficacia se refiere a la capacidad percibida y su implicación puede ser observada tanto a nivel comportamental, como en metas y aspiraciones, expectativas de resultados y en la percepción de impedimentos u oportunidades en el entorno social ${ }^{3}$. La autoeficacia tiene como elemento central las percepciones que tienen los individuos de su capacidad de actuar. Desde la perspectiva de la teoría social cognitiva, la percepción de autoeficacia es uno de los factores personales que determinan la motivación y la ejecución ${ }^{4}$.

Además, la teoría social cognitiva explica cómo la autoeficacia puede ser una estrategia para integrar una nutrición saludable en el estilo de vida ${ }^{5-7}$. Las creencias sobre las barreras percibidas pueden ser un predictor del manejo de la dieta, ya que se asocian con menos comportamientos saludables en personas con riesgo de $\mathrm{SM}^{8}$. Así, los sujetos con mayor confianza en su capacidad para tomar decisiones saludables ingirieron menos grasa y más fibras, frutas y verduras $^{6,9}$. Intervenciones enfocadas en la autoeficacia para el consumo de frutas y verduras, también demostraron un aumento en la ingesta de ambas en 6 meses de seguimiento ${ }^{10}$.

En esta misma línea, se observó que la obesidad y el índice de masa corporal (IMC) se asocian con baja autoeficacia y alimentación inadecuada. De modo que una mayor autoeficacia y metas claras para una alimentación saludable, fueron optimizadas en intervenciones para el cambio del estilo de vida en pacientes obesos ${ }^{11}$. Asimismo, los sujetos que lograron menos pérdida de peso presentaron disminuciones en la autoeficacia del comportamiento alimentario que se asociaron con la recuperación de peso en el largo plazo ${ }^{12}$. Por otro lado, pacientes con mayor autoeficacia dietética tuvieron menor aumento de peso y comportamientos de cum- 
plimiento favorables hacia las dietas prescritas, con efectos beneficiosos sobre la salud física y la calidad de vida ${ }^{13}$.

Aunque que se haya demostrado la importancia de la autoeficacia en el comportamiento saludable, pocas escalas están validadas en este contexto. El "Inventario de Autoeficacia para el Control de Peso", compuesto por 37 ítems, evalúa el estilo de alimentación, la influencia de los estímulos externos en la conducta de sobreingesta y los patrones de actividad física; el instrumento demostró una estructura de 3 factores con consistencia interna satisfactoria ${ }^{14}$. El mismo inventario fue adaptado en un estudio realizado con adolescentes mexicanos, con versión para el sexo masculino y el femenino, y demostró ser adecuado para evaluar la autoeficacia"15. La "Escala de autoeficacia de la conducta alimentaria" compuesta por 21 ítems, que evalúan la capacidad percibida para disminuir la ingesta de productos calóricos o dulces y aumentar el consumo de alimentos saludables también demostró fiabilidad adecuada ${ }^{16}$.

En esta línea, verificamos el contenido de los 30 ítems de la "Escala de Autoeficacia para los Hábitos Alimentarios" (AEHA), desarrollada por Bandura ${ }^{5}$, pero sin estudios de validación por parte del autor, y la elegimos de acuerdo con los objetivos del estudio "Programa de Intervención Multimodal para pacientes con Síndrome Metabólico - PROMETS" registrado en www.clinicaltrials.gov (NCT02949622). Los resultados esperados se refieren a que, concomitante al aumento de la autoeficacia, se observa un aumento en los comportamientos relacionados a la alimentación saludable en pacientes con síndrome metabólico. Con respecto a estudios previos realizados con esta escala, se hallaron las propiedades psicométricas (solución de 3 factores) en sujetos con al menos un factor de riesgo cardiovascular (Brasil) ${ }^{17}$. Sin embargo, no encontramos ningún estudio de validación de las escalas de autoeficacia de Bandura en la población de habla hispana, por ello también hemos Ilevado a cabo la validación de la "Escala de autoeficacia para el ejercicio físico en la misma población clínica"18. Considerando el papel de la autoeficacia en el cambio del estilo de vida, especialmente en la alimentación saludable, el objetivo del estudio es describir, preliminarmente, las propiedades psicométricas la AEHA (análisis factorial, de la consistencia interna, estabilidad temporal, sensibilidad al cambio y validez concurrente) en pacientes con SM en España y aportar informaciones sobre su uso en contextos clínicos. La muestra clínica elegida, igualada en la línea base en cuanto a los factores sociodemográficos, ha permitido la realización de distintos análisis psicométricos para evaluar la fiabilidad y la validez de la escala. Conjuntamente, hemos comprobado la sensibilidad del instrumento como medida pre y postratamiento en una intervención cognitivo conductual para pacientes con SM.

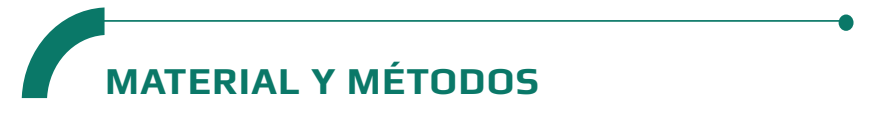

\section{Participantes}

Participaron en este ensayo clínico aleatorizado 135 sujetos (25 a 65 años), diagnosticados con SM. Los criterios de inclusión y diagnóstico fueron de acuerdo con el ATP-III ${ }^{1}$. Los criterios de exclusión fueron diagnósticos de artrosis avanzada, enfermedades inflamatorias activas, presencia de deterioro cognitivo ${ }^{19}$ y no saber leer ni escribir. Todos los sujetos firmaron el consentimiento informado elaborado de acuerdo a las recomendaciones de la Declaración de Helsinki y la investigación fue aprobada por el Comité Ético del Hospital Universitario Virgen de las Nieves (HUVN).

\section{Medidas}

Entrevista de datos sociodemográficos y de estilo de vida: Es una entrevista estructurada construida ad hoc que permite obtener información sobre datos sociodemográficos, actividad física, alimentación y consumo de alcohol y tabaco. En lo que respecta a los datos sociodemográficos (p. ej., sexo, edad, estado civil, escolarización, si ejercía actividad laboral), éstos son utilizados para establecer un perfil de la muestra evaluada. La entrevista incluye preguntas que se refieren a la práctica de actividad física: tipo de práctica, frecuencia (en la semana), por cuánto tiempo, así como sobre la percepción de alimentación, si estaba adecuada o necesitaba cambios. Por último, para evaluar el consumo de alcohol se preguntó sobre la ingesta, el tipo de bebida, la frecuencia y la dosis habitualmente ingerida y si eran fumadores.

Prueba de Fagerström para dependencia de nicotina: Consta de 6 ítems con dos o cuatro alternativas de respuesta. La puntuación oscila entre 0 y 10 . Puntuaciones altas en el FTND (6 o más) indican un alto grado de dependencia, mientras que puntuaciones bajas no necesariamente indican un bajo grado de dependencia. Esta escala es el instrumento más utilizado para evaluar la dependencia de la nicotina, aunque su fiabilidad con muestra española es moderada (alfa de Cronbach $=0,65)^{20}$.

Cuestionario de Adherencia a la Dieta Mediterránea: Fue la medida utilizada para evaluar el nivel de adherencia a la Dieta Mediterránea (DM). El grado de adherencia a la DM está determinado por la suma de los ítems según los puntajes del participante. Cada ítem se refiere al consumo de un alimento en particular, reflejando un comportamiento autoinformado. Una puntuación inferior a 11 se considera de baja adherencia, mientras que una puntuación de 11 o más es de alta adherencia21. 
Escala de Autoeficacia para los Hábitos Alimentarios-AEHA (Self-Efficacy to Regulate Eating Habits-SEREH) ${ }^{5}$ : Registra el grado de confianza para mantener una alimentación saludable en situaciones difíciles de hacerlo. Cuanto más alta la puntuación mayor la autoeficacia (oscila entre 0 y 100). Para la versión final del cuestionario, se utilizó el procedimiento de traducción del inglés al español y retrotraducción del español al inglés hasta lograr un acuerdo entre los traductores.

Inventario de Expresión de Ira Estado-Rasgo-STAXI-2: Evalúa facetas de la ira como estado y rasgo. Consta de 49 ítems organizados en 6 escalas y 5 subescalas, permitiendo obtener un índice de cada escala y subescala, así como un índice general del inventario. La consistencia interna de las escalas y las subescalas medidos con el alfa de Cronbach varía entre 0,67 y $0,89^{22}$.

Inventario de Asertividad23: Evalúa la asertividad general. Consta de 30 ítems divididos en dos subescalas:

(1) Grado de malestar (GM): grado de ansiedad que provoca en el sujeto diversas situaciones sociales.

(2) Probabilidad de respuesta (PR): la probabilidad estimada de que una persona manifieste una conducta asertiva específica.

La puntuación total para ambas subescalas oscila entre 40 y 200, con mayores puntuaciones indicando menor asertividad. El inventario, en su versión original, tenía una buena estabilidad temporal ( 5 semanas) para ambas subescalas, con coeficientes de correlación de Pearson de $r=0,87$ para la subescala GM y r=0,81 para la subescala PR. Además, el inventario ha revelado buena consistencia interna con coeficientes alfa de Cronbach de 0,91 y 0,92 para la subescala de $\mathrm{GM}^{24,25}$ y de 0,87 a 0,94 para la subescala $\mathrm{PR}^{24,26}$.

Escala de Estrés Percibido: Consta de 14 ítems que evalúan el estrés percibido en el último mes. Fue diseñada para medir el grado de estrés en las diversas situaciones que puedan ocurrir en la vida. La versión española de la escala demostró una adecuada fiabilidad (consistencia interna, $\alpha=0,81$; y test-retest, $r=0,73)^{27}$.

Cuestionario de Asertividad Centrado en el Estilo de VidaCACEV: Se compone de 18 ítems que miden el grado de malestar, tensión o nerviosismo en situaciones de interacción social relacionadas con un estilo de vida saludable (mantener una alimentación saludable y la práctica regular de ejercicio físico). Cuanto más elevada sea la puntuación menor será la asertividad. EI CACEV demostró una adecuada fiabilidad (consistencia interna, $\alpha=0,925)$, validez (convergente), sensibilidad y estabilidad temporal ${ }^{28}$.
Datos antropométricos como peso y talla fueron evaluados para verificar el IMC y la circunferencia de la cintura, medida utilizando una cinta métrica colocada en un plano horizontal, situado en el punto medio entre la última costilla y la cresta iliaca. Datos clínicos, como la presión arterial, verificada de acuerdo con la American Heart Association², el perfil lipídico y la glucemia en ayunas fueron recolectados.

\section{Procedimiento}

Se llevó a cabo, en primer lugar, una entrevista estructurada con los participantes, por medio de la cual se obtuvieron las principales variables sociodemográficas. Además, se tomaron las medidas antropométricas, se solicitaron los análisis bioquímicos y se aplicaron los instrumentos de evaluación. El procedimiento completo tuvo una duración de aproximadamente 2 horas.

Para verificar la sensibilidad al cambio del instrumento, los sujetos participaron en una intervención para el cambio del estilo de vida y fueron distrubuidos al azar en dos grupos: grupo experimental (GE) y grupo control (GC). El programa de intervención del GE estaba basado en la terapia cognitivo conductual y fue realizado en grupos, compuestos por 10 a 12 pacientes por grupo y con una duración de 12 sesiones semanales de 90 minutos de duración. Cada sesión se centraba en un tema diferente en el programa de terapia cognitivo conductual (TCC), como la modificación de creencias o pensamientos disfuncionales, el manejo del estrés, el control de la ira, el entrenamiento en asertividad o en habilidades sociales. El objetivo de esta intervención fue aportar información sobre la enfermedad, así como dotar al paciente de estrategias cognitivas y conductuales tanto para el cambio de estilo de vida como para la adherencia a las medidas terapéuticas propuestas. EI GC asistió a una charla con informaciones básicas sobre el riesgo cardiovascular (medidas terapéuticas estándar que consisten en hábitos alimentarios saludables y actividad física) ${ }^{29}$. La duración de esta "intervención" fue de 90 minutos en una única ocasión por grupo, cada uno de ellos compuestos por, aproximadamente, 10 a 15 personas.

Los pacientes fueron asignados aleatoriamente (1:1) al GE (TCC) o al GC. La aleatorización fue generada por computadora con bloques de seis, estratificados por tipo de inscripción (tipo de consulta). Un asistente no involucrado en el ensayo hizo la aleatorización en presencia del paciente y el investigador. Debido a la naturaleza del estudio, los pacientes no fueron cegados al tipo de tratamiento que recibieron (TCC vs. charla) pero cegados al grupo al que habían sido asignados, y el investigador no fue cegado por razones prácticas. El investigador que obtuvo los datos de referencia no 
estuvo cegado a la asignación del grupo del sujeto, pero el investigador que realizó la evaluación posterior al tratamiento y la evaluación de seguimiento estaba cegado a la asignación del grupo. Un investigador independiente no involucrado en el estudio, que también estaba cegado a la asignación de grupo, realizó el análisis estadístico.

Las medidas fueron evaluadas en 4 momentos para ambos los grupos: T0=línea base (pretratamiento); T1=postratamiento; T2=seguimiento de 6 meses y T3=seguimiento de 18 meses, con el objetivo de evaluar la sensibilidad al cambio del instrumento a lo largo del seguimiento. El total de participantes al final del seguimiento fue $\mathrm{GE}=48$ sujetos y $\mathrm{GC}=30$.

\section{Determinación del tamaño de la muestra}

El número mínimo de participantes para la realización del análisis factorial exploratorio (AFE) puede variar. De Winter, Dodou y Wieringa ${ }^{30}$ citan reglas en relación a la muestra mínima total (variante de 50 hasta 1.000 participantes) y directrices de muestra mínima proporcional de sujeto en relación al número de ítems (3:1, 5:1, 20:1). Sin embargo, tales "reglas de oro" se mostraron, a lo largo del tiempo, inefectivas. Por ello, es necesario considerar las comunalidades entre ítems y factores, número de factores y número de ítems en cada factor ${ }^{31}$. MacCallum et al. ${ }^{31}$ propusieron que para comunalidades superiores al 60\%, la muestra ideal sería de aproximadamente 100 individuos. En este estudio, la comunalidad media de la AEAH fue alta $(71,20)$, lo que sugiere un número mínimo de 100 participantes para realizar el AFE.

\section{Análisis estadísticos}

Análisis descriptivos fueron realizados para la caracterización de las muestras. Pruebas t de Student y Chi-Cuadrado de Pearson para verificar posibles diferencias significativas entre los grupos (experimental y control) en variables sociodemográficas, antropométricas y de riesgo cardiovascular, respecto a la línea base. La normalidad fue evaluada con valores de asimetría/Skewness (Sk>|3|) y curtosis/Kurtosis $(\mathrm{Ku}>|10|)$, indican graves violaciones a la distribución normal.

La adecuación de la matriz de correlaciones fue analizada con la prueba de esfericidad de Bartlett, cuyo valor significativo $(p \leq 0,05)$ indica que la matriz de datos presenta correlaciones significativas entre las variables ${ }^{32}$. La adecuación de los datos para la extracción de factores fue evaluada con la Medida de Adecuación de la Muestra de Kaiser-Meyer-Olkin$\mathrm{KMO}$, la cual varía de 0 a 1 , siendo inaceptable $(<0,50)$, malo $[0,50-0,59]$, regular $[0,60-0,69]$, aceptable $[0,70-0,79]$, bueno $[0,80-0,89]$ o excelente $(\geq 0,90)$. El análisis paralelo y el AFE fueron utilizados para análisis y extracción de los factores, con rotación oblicua Promax (esta rotación permite que los factores estén correlacionados) ${ }^{32}$ y normalización de Kaiser. Para la retención de los factores fueron considerados los criterios de Kaiser (valor propio $\geq 1,00$ ) y de Catell (gráfico de sedimentación). Saturaciones factoriales $\geq 0,40$ fueron consideradas como criterio de saturación de los ítems en cada factor. Fueron excluidos: Ítems que saturaron simultáneamente en más de un factor; Ítems que no contribuyan (o que disminuyan) la consistencia interna del factor, de acuerdo con los valores del alfa si el ítem es eliminado ${ }^{32}$; Ítems con comunalidades <0,40; Valores correlación ítem-total corregidos $\leq 0,30^{32}$.

El análisis de la consistencia interna del AEHA se evaluó con el coeficiente alfa de Cronbach (valores >0,70 son considerados adecuados) y la fiabilidad con la fórmula de las dos mitades de Guttman. También se realizaron correlaciones de Pearson o Spearman (nivel de significación de 5\%) entre la cantidad de comidas al día, la percepción de una alimentación saludable, la capacidad percibida para renunciar a un alimento no saludable, la adherencia a la Dieta Mediterránea, la asertividad general y específica al estilo de vida, la ira y el estrés.

El análisis factorial, la consistencia interna del AEHA y las correlaciones con otras variables fueron realizados con datos de la muestra total (135 sujetos: 77 del GE y 58 del GC) evaluados en el TO.

Por último, se realizaron pruebas de t de Student para verificar las diferencias entre e intra grupos en los distintos momentos de evaluación, en relación a la estabilidad temporal y a la sensibilidad al cambio. Los análisis fueron realizados en el programa SPSS (versión 21).

\section{RESULTADOS}

Las características sociodemográficas, datos antropométricos y factores de riesgo cardiovascular de la muestra analizada están descritos en la Tabla 1.

\section{Análisis de datos preliminares}

Los valores de asimetría y curtosis no indicaran violaciones graves a la normalidad ( $\mathrm{Sk} \leq 2,10$ y $\mathrm{Ku} \leq 8,22)$. Análisis preliminares del conjunto inicial de 30 ítems de la escala indicaron una prueba de esfericidad de Bartlett significativa $\left(p<0,001 ; \chi^{2}=3.698,103 ; d f=425\right)$, con una excelente KMO $(0,946)$, revelando que los datos son adecuados para extracción de factores.

acción de factores. 
Rev Esp Nutr Hum Diet. 2019; 23(1): 14 - 26

Propiedades psicométricas de la escala de autoeficacia para los hábitos alimentarios (AEHA)

Tabla 1. Características descriptivas de la muestra de pacientes con síndrome metabólico en línea base.

\begin{tabular}{|c|c|c|c|c|}
\hline Variables & Total $(n=135)$ & GE $(n=77)$ & $G C(n=58)$ & p-valor \\
\hline \multicolumn{5}{|c|}{ SOCIODEMOGRÁFICAS } \\
\hline Edad, M (DT) & $55,5(7,6)$ & $55,1(8,4)$ & $55,9(6,4)$ & 0,559 \\
\hline $\begin{array}{l}\text { Sexo, } \mathbf{n}(\%) \\
\text { Femenino } \\
\text { Masculino }\end{array}$ & $\begin{array}{l}50,4(68) \\
49,6(67)\end{array}$ & $\begin{array}{l}39(50,6) \\
38(49,4)\end{array}$ & $\begin{array}{l}29(50) \\
29(50)\end{array}$ & 0,940 \\
\hline $\begin{array}{l}\text { Estado Civil, n (\%) } \\
\text { Soltero } \\
\text { Casado } \\
\text { Otros }\end{array}$ & $\begin{array}{l}14,8(20) \\
77(104) \\
8,1(11)\end{array}$ & $\begin{array}{c}10(13) \\
79,2(61) \\
7,8(6)\end{array}$ & $\begin{array}{c}17,2(10) \\
74,1(43) \\
8,6(5)\end{array}$ & 0,171 \\
\hline $\begin{array}{l}\text { Educación, n (\%) } \\
\text { Básica } \\
\text { Bachiller } \\
\text { Ciclo medio } \\
\text { Universitaria }\end{array}$ & $\begin{array}{c}40,5(53) \\
13,7(18) \\
26,7(35) \\
19(25)\end{array}$ & $\begin{array}{l}37,7(29) \\
14,3(11) \\
22,1(17) \\
20,8(16)\end{array}$ & $\begin{array}{l}41,3(24) \\
12,1(7) \\
31(18) \\
15,5(9)\end{array}$ & 0,632 \\
\hline $\begin{array}{l}\text { Trabajo, n (\%) } \\
\text { Sí } \\
\text { No }\end{array}$ & $\begin{array}{l}46,6(62) \\
53,4(71)\end{array}$ & $\begin{array}{l}46,7(35) \\
53,3(40)\end{array}$ & $\begin{array}{l}46,6(27) \\
53,4(31)\end{array}$ & 0,989 \\
\hline $\begin{array}{l}\text { Ejercicio Físico, } \mathbf{n} \text { (9) } \\
\text { Sí } \\
\text { No }\end{array}$ & $\begin{array}{l}65,9(89) \\
34,1(46)\end{array}$ & $\begin{array}{l}59,7(46) \\
40,3(31)\end{array}$ & $\begin{array}{l}74,1(43) \\
25,9(15)\end{array}$ & 0,081 \\
\hline $\begin{array}{l}\text { Alimentación, n (\%) } \\
\text { Adecuada } \\
\text { Necesita cambios }\end{array}$ & $\begin{array}{l}35,6(48) \\
64,5(87)\end{array}$ & $\begin{array}{l}33,8(26) \\
66,2(51)\end{array}$ & $\begin{array}{l}37,9(22) \\
62,1(36)\end{array}$ & 0,412 \\
\hline $\begin{array}{l}\text { Tabaco, n (\%) } \\
\text { Sí } \\
\text { No }\end{array}$ & $\begin{array}{c}22,2(30) \\
77,8(105)\end{array}$ & $\begin{array}{l}26(20) \\
74(57)\end{array}$ & $\begin{array}{l}17,2(10) \\
82,8(48)\end{array}$ & 0,227 \\
\hline $\begin{array}{l}\text { Alcohol, n (\%) } \\
\text { Sí } \\
\text { No }\end{array}$ & $\begin{array}{l}60(81) \\
40(54)\end{array}$ & $\begin{array}{c}62,32(48) \\
37,7(27)\end{array}$ & $\begin{array}{l}56,9(33) \\
43,1(25)\end{array}$ & 0,523 \\
\hline
\end{tabular}

VARIABLES ANTROPOMÉTRICAS Y FACTORES DE RC

\begin{tabular}{l|c|c|c|c}
\hline \begin{tabular}{l|l|l} 
Peso, M (DT) \\
Mujer
\end{tabular} & & & \\
Hombre & $81,0(13,0)$ & $84,1(13,6)$ & $76,9(11,1)$ & $0,024^{*}$ \\
\hline IMC, M (DT) & $96,4(14,4)$ & $99,1(14,5)$ & $92,8(13,8)$ & 0,077 \\
\hline $\begin{array}{l}\text { Mujer } \\
\text { Hombre }\end{array}$ & $32,73(5,14)$ & $34,3(5,4)$ & & \\
\hline
\end{tabular}




\begin{tabular}{|c|c|c|c|c|}
\hline Variables & Total ( $n=135)$ & GE (n=77) & GC $(n=58)$ & $\mathrm{p}$-valor \\
\hline \multicolumn{5}{|c|}{ VARIABLES ANTROPOMÉTRICAS Y FACTORES DE RC } \\
\hline \multicolumn{5}{|l|}{ CA, M (DT) } \\
\hline Mujer & $108,0(12,0)$ & $111,8(12,3)$ & $102,9(9,4)$ & $0,002^{* *}$ \\
\hline Hombre & $113,1(9,94)$ & $116,2(9,5)$ & $110,9(9,8)$ & $0,031^{*}$ \\
\hline PAS, M (DT) & $133,9(19,9)$ & $135,0(19,2)$ & $132,4(20,9)$ & 0,468 \\
\hline PAD, M (DT) & $86,4(12,7)$ & $87,7(13,6)$ & $84,7(11,5)$ & 0,193 \\
\hline Colesterol, M (DT) & $193,7(45,5)$ & $201,0(45,3)$ & $183,2(44,1)$ & $0,031^{*}$ \\
\hline \multicolumn{5}{|l|}{ HDLc, M (DT) } \\
\hline Mujer & $51,9(12,7)$ & $54,5(12,9)$ & $48,0(11,4)$ & 0,048 \\
\hline Hombre & $42,0(13,0)$ & $41,5(12,4)$ & $42,6(14,1)$ & 0,767 \\
\hline LDLc, M (DT) & $117,8(42,0)$ & $123,4(43,5)$ & $109,8(38,9)$ & 0,078 \\
\hline Triglicéridos, M (DT) & $191,2(101,8)$ & $199,4(111,4)$ & $179,3(85,9)$ & 0,282 \\
\hline Glucemia, M (DT) & $118,0(37,9)$ & $120,6(43,2)$ & $114,2(28,8)$ & 0,359 \\
\hline
\end{tabular}

GE: grupo experimental; GC: grupo control; M: media; DT: desviación típica; RC: riesgo cardiovascular; IMC: índice de masa corporal; CA: circunferencia abdominal; PAS: presión arterial sistólica; PAD: presión arterial diastólica; HDLc: lipoproteínas de alta densidad; LDLc: lipoproteínas de baja densidad; ${ }^{*} \mathbf{p} \leq 0,05 ; * * \mathbf{p} \leq 0,01$.

\section{Análisis factorial exploratorio de la AEHA}

Inicialmente, los 30 ítems que componen la escala fueron sometidos a un análisis paralelo en el programa JASP, que indicó la existencia de dos factores $\left(\chi^{2}=724,10\right.$; $d f=376, p<0,001)$. A continuación, fue realizado un AFE con el metodo de la máxima verosimilitud, en el cual se fijó el número de factores en 2 , y se utilizó la rotación Promax. En la Tabla 2 presentamos la solución factorial extraída y las saturaciones de cada ítem. Considerando la matriz rotada, la solución de 2 factores explica el 60,97\% de la varianza común. El primer factor, F1: "Situaciones sociales o estímulo externo para disfrutar de la comida" tiene un valor proprio de 16,94 y explica el $56,48 \%$ de la varianza común. El segundo factor $\mathrm{F} 2$ : "Sentimientos negativos" tiene un valor proprio de 2,04 y explica el 6,81\% de la varianza común.

Con base en el análisis de los factores y respectivos ítems, comunalidades y cargas factoriales, excluimos el ítem 6 del factor 1 y el ítem 3 del factor 2 porque saturaron en los dos factores y los ítems 1 y 9 por puntuar más alto que el alfa total del factor 2 en el alfa si el ítem es eliminado ${ }^{22}$. Así, la escala total de 26 ítems presenta una solución de 2 factores, siendo el primero compuesto por 21 ítems y el segundo por 5 ítems.

\section{Análisis de fiabilidad}

La versión final de 26 ítems del AEHA demostró valores de consistencia interna de adecuados a excelentes para el F1 $(0,969$ y 0,962), F2 $(0,935$ y 0,887) y para la puntuación total $(0,971$ y 0,957$)$, segundo los valores del alfa de Cronbach y del método de las dos mitades de Guttman, respectivamente. Además, segundo los valores de alfa si el ítem es eliminado, fue posible observar que todos los ítems estaban contribuyendo para la consistencia interna observada en cada uno de los 2 factores (F1: $\alpha \leq 0,931 ; F 2: \alpha \leq 0,969$ ).

La fiabilidad (alfa de Cronbach y método de las dos mitades de Guttman) también fue verificada en cada grupo antes y después de la intervención (fiabilidad test-retest). Los valores para el GE pre intervención se presentan a continuación en ambos los métodos: alfa de Cronbach ( $F 1=0,972 ; F 2=0,949$ y total $=0,975)$ y dos mitades de Guttman ( $F 1=0,969$; $F 2=0,901$ y total $=0,946)$, y para el $G C(F 1=0,963$; $F 2=$ $0,914$ y total $=0,965)$ y dos mitades de Guttman ( $F 1=0,952$; $F 2=0,874$ y total $=0,933$ ). Los valores para el GE post intervención se presentan a continuación en ambos los métodos: alfa de Cronbach ( $F 1=0,970 ; F 2=0,941$ y total $=0,972)$ y dos mitades de Guttman ( $F 1=0,948 ; F 2=0,952$ y total $=0,956$ ); $y$ para el GC ( $F 1=0,976 ; F 2=0,924$ y total $=0,981)$ y dos mitades de Guttman ( $F 1=0,980 ; F 2=0,926$ y total $=0,972$ ). 
Rev Esp Nutr Hum Diet. 2019; 23(1): 14 - 26

Propiedades psicométricas de la escala de autoeficacia para los hábitos alimentarios (AEHA)

Tabla 2. Análisis factorial exploratoria para la extracción de los factores de la escala AEHA, con la saturación de los ítems de acuerdo con la matriz de rotación Promax.

Ítems de cada factor

№

Promax

F1 $:$ F2

FACTOR 1: SITUACIONES SOCIALES O ESTÍMULO EXTERNO PARA DISFRUTAR DE LA COMIDA

(Eigenvalue: 16,94; Porcentaje de varianza explicada: 56,48\% y Alpha de Cronbach: 0,969)

En las fiestas donde se sirven una gran cantidad de rica comida alta en grasas.

Fiestas y celebraciones donde se sirve comida alta en grasas.

En eventos de ocio y deportivos en los que se sirven comidas rápidas altas en grasa.

19

$1,012:-0,169$

Comiendo fuera con los demás cuando ellos han pedido comidas altas en grasas.

Cuando visito una ciudad y quiero disfrutar de la comida y los restaurantes locales.

\begin{tabular}{l|l|l}
24 & 0,937 & $-0,078$
\end{tabular}

Cuando siento un fuerte deseo de comer alimentos altos en grasa que me gustan.

\begin{tabular}{l|l:l}
20 & 0,918 & $-0,077$
\end{tabular}

Cuando otros traen o sirven comida alta en grasas.

Cenando en la casa de un amigo.

Comidas de avión con alimentos altos en grasa.

Alguien me ofrece alimentos altos en grasa.

$18 \quad 0,845:-0,045$

Cuando me encuentro con comida apetitosa alta en grasas en el supermercado.

\begin{tabular}{l|l|l|l}
23 & 0,791 & $-0,002$
\end{tabular}

Celebrándolo con los demás.

\begin{tabular}{l|l|l}
15 & 0,788 & 0,088
\end{tabular}

Al visitar una ciudad y necesitar una comida rápida.

Cuando en casa hay mucha comida alta en grasas.

Cuando estoy entreteniendo a las visitas.

Cuando quiero algo de variedad en mi dieta.

Durante las vacaciones.

\begin{tabular}{l|l|l}
28 & 0,766 & 0,114
\end{tabular}

\begin{tabular}{l|l|l}
5 & $\mathbf{0 , 7 5 0}$ & $-0,052$
\end{tabular}

\begin{tabular}{l|l|l}
22 & $\mathbf{0 , 7 4 7}$ & 0,048
\end{tabular}

Al desayunar en un restaurante.

Cuando tengo que preparar mis propias comidas.

\begin{tabular}{l|l|l}
14 & 0,737 & 0,033
\end{tabular}

Cuando quiero algo de variedad en mi dieta.

\begin{tabular}{l|l|l}
30 & $\mathbf{0 , 7 1 1}$ & 0,084
\end{tabular}

\begin{tabular}{l|l|l}
13 & 0,703 & 0,017
\end{tabular}

Comiendo solo en un restaurante.

Preparando comida para otras personas.

\begin{tabular}{l|l|l}
21 & 0,700 & 0,107
\end{tabular}

\begin{tabular}{l|l|l}
12 & 0,681 & 0,185
\end{tabular}

\begin{tabular}{l|l|l}
16 & $\mathbf{0 , 5 8 6}$ & 0,201
\end{tabular}

\begin{tabular}{l|l|l}
11 & $\mathbf{0 , 5 0 9}$ & 0,353
\end{tabular}

\begin{tabular}{l|l|l}
17 & $\mathbf{0 , 4 8 3}$ & 0,302
\end{tabular}

\begin{tabular}{l|l|l|l}
27 & $\mathbf{0 , 4 7 3}$ & 0,331
\end{tabular}

\begin{tabular}{l|l|l}
29 & 0,470 & 0,277
\end{tabular}

\begin{tabular}{l|l|l}
26 & $\mathbf{0 , 4 6 9}$ & 0,328
\end{tabular}

\begin{tabular}{l|l}
7 & 0,469
\end{tabular}

0,188

$6^{*}$

0,394

0,313

\section{FACTOR 2: SENTIMIENTOS NEGATIVOS}

(Eigenvalue: 2,04; Porcentaje de varianza explicada: 6,81\% y Alpha de Cronbach: 0,935)

Cuando estoy enfadado o molesto.

Cuando estoy deprimido.

Cuando siento malestar o tensión con respecto a asuntos relacionados con el trabajo.

Cuando estoy molesto por cuestiones familiares.

Sintiéndome inquieto o aburrido.

Cuando tengo mucha hambre.

Mientras veo la televisión.

En los días festivos.

\begin{tabular}{c:c|c}
\hline 8 & $-0,193$ & $\mathbf{1 , 0 8 0}$ \\
\hline 10 & $-0,062$ & $\mathbf{0 , 9 2 2}$ \\
\hline 4 & $-0,080$ & $\mathbf{0 , 8 6 8}$ \\
\hline 25 & 0,041 & $\mathbf{0 , 8 0 0}$ \\
\hline 2 & 0,136 & $\mathbf{0 , 7 3 7}$ \\
\hline $9^{*}$ & 0,201 & $\mathbf{0 , 5 6 4}$ \\
\hline $1^{*}$ & 0,250 & $\mathbf{0 , 4 4 5}$ \\
\hline $3^{*}$ & 0,306 & $\mathbf{0 , 3 3 9}$ \\
\hline
\end{tabular}




\section{Diferencias entre grupos y validez externa}

Las medias y desviaciones típicas (línea base) de ambos grupos están descritas en la Tabla 3. Los análisis de correlación entre la AEHA y las variables psicológicas y de estilo de vida están en la Tabla 4. Estas variables fueron elegidas para la validez externa ya que se refieren a comportamientos específicos que, de acuerdo con la literatura, están asociados a la autoeficacia (percepción de una alimentación saludable, la capacidad percibida para renunciar a un alimento no saludable, la adherencia a la Dieta Mediterránea) ${ }^{6,9-11}$. En cuanto a la asertividad general y específica al estilo de vida, la ira y el estrés, las mismas variables se asociaron con la autoeficacia en el ejercicio en la misma población clínica ${ }^{18}$. Hallamos también las correlaciones entre los dos factores de la escala, $(r=0,70, p<0,001)$. F1 y total $(r=0,98, p<0,001)$ y $F 2$ y total $(r=0,81, p<0,001)$.

\section{Sensibilidad al cambio y estabilidad temporal}

Se realizaron pruebas $t$ (muestras independientes) para explorar diferencias en la puntuación de los resultados de la escala y sus dos factores entre los participantes del GE y GC a los 3, 6 y 18 meses. Hemos encontrado diferencias a los 6 meses en el $F 2\left(t_{(76)}=1,93, p=0,028\right)$, siendo que el $G E$ presentó medias superiores al GC.

En cuanto a las diferencias intra grupos, en la prueba t para muestras emparejadas en el GE hemos encontrado diferencias significativas en la puntuación total a los $3\left(\mathrm{t}_{(47)}=-1,99\right.$,

Tabla 3. Medias y desviaciones típicas en la puntuación total y en los factores de la AEHA en ambos los grupos.

\begin{tabular}{|c|c|c|c|c|c|c|c|c|}
\hline \multirow{2}{*}{ Variables } & \multicolumn{4}{|c|}{$\begin{array}{c}\mathrm{GE}(\mathrm{n}=48) \\
M(D T)\end{array}$} & \multicolumn{4}{|c|}{$\begin{array}{c}\mathrm{GC}(\mathrm{n}=30) \\
M(D T)\end{array}$} \\
\hline & TO & $\mathrm{T} 1$ & $\mathrm{~T} 2$ & T3 & TO & T1 & $\mathrm{T} 2$ & T3 \\
\hline AEHA T & $\begin{array}{c}57,9 \\
(26,1)\end{array}$ & $\begin{array}{c}64,8 \\
(20,7)\end{array}$ & $\begin{array}{c}64,0 \\
(21,3)\end{array}$ & $\begin{array}{c}61,8 \\
(22,2)\end{array}$ & $\begin{array}{c}57,4 \\
(20,9)\end{array}$ & $\begin{array}{c}58,2 \\
(23,0)\end{array}$ & $\begin{array}{c}60,6 \\
(26,0)\end{array}$ & $\begin{array}{l}59,9 \\
(23,1)\end{array}$ \\
\hline AEHA F1 & $\begin{array}{c}57,6 \\
(26,8)\end{array}$ & $\begin{array}{c}64,0 \\
(22,5)\end{array}$ & $\begin{array}{c}60,1 \\
(24,1)\end{array}$ & $\begin{array}{c}61,3 \\
(22,3)\end{array}$ & $\begin{array}{c}57,9 \\
(21,0)\end{array}$ & $\begin{array}{c}56,7 \\
(24,8)\end{array}$ & $\begin{array}{c}51,2 \\
(27,5)\end{array}$ & $\begin{array}{c}53,2 \\
(25,5)\end{array}$ \\
\hline AEHA F2 & $\begin{array}{c}59,0 \\
(30,5)\end{array}$ & $\begin{array}{c}64,3 \\
(23,7)\end{array}$ & $\begin{array}{c}62,8 \\
(24,0)\end{array}$ & $\begin{array}{c}60,6 \\
(25,2)\end{array}$ & $\begin{array}{c}55,6 \\
(25,5)\end{array}$ & $\begin{array}{c}55,3 \\
(26,4)\end{array}$ & $\begin{array}{c}51,6 \\
(26,3)\end{array}$ & $\begin{array}{c}52,7 \\
(25,0)\end{array}$ \\
\hline
\end{tabular}

GE: grupo experimental; GC: grupo control; M: media; DT: desviación típica; AEHA T: puntuación total; AEHA F1: Factor 1; AEHA F2: Factor 2.

Tabla 4. Correlación entre la AEHA y las demás variables psicológicas y de estilo de vida.

\begin{tabular}{lcccccccccccc} 
Variables & FC & DS & ANS & DM & AI-ME & Al-PR & STAXI & PSS & Ct & Cf1 & Cf2 & Cf3 \\
\hline AEHA T & $0,20^{*}$ & $0,23^{* *}$ & $0,35^{* *}$ & $0,20^{*}$ & $-0,26^{* *}$ & $-0,28^{* *}$ & $-0,25^{* *}$ & $-0,16$ & $-0,27^{* *}$ & $-0,22^{* *}$ & $-0,28^{* *}$ & $-0,18^{*}$ \\
\hline AEHA F1 & $0,24^{*}$ & $0,24^{* *}$ & $0,37^{* *}$ & $0,19^{*}$ & $-0,25^{* *}$ & $-0,27^{* *}$ & $-0,24^{* *}$ & $-0,11$ & $-0,25^{* *}$ & $-0,21^{*}$ & $-0,28^{* *}$ & $-0,17^{*}$ \\
\hline AEHA F2 & 0,05 & 0,14 & $0,20^{*}$ & $0,20^{*}$ & $-0,24^{* *}$ & $-0,28^{* *}$ & $-0,23^{* *}$ & $-0,28^{* *}$ & $-0,24^{* *}$ & $-0,22^{*}$ & $-0,20^{*}$ & $-0,19^{*}$
\end{tabular}

AEHA T: puntuación total; AEHA F1: Factor 1 - Situaciones sociales o estímulo externo para disfrutar de la comida;

AEHA F2: Factor 2 - Sentimientos negativos; FC: frecuencia de comidas al día; DS: dieta saludable; ANS: alimento no saludable; DM: Dieta Mediterránea; AI-ME: Inventario de Asertividad - Mal estar; AI-PR: Inventario de Asertividad - Probabilidad de respuesta; STAXI: índice de expresión de la ira; PSS: escala de estrés percibido; Ct: puntuación total CACEV; Cf1: CACEV F1 - Expresividad general (mantener alimentación saludable y práctica de ejercicio físico); Cf2: CACEV F2 - Decir que no relacionado con el consumo de alimentos poco saludables; Cf3: CACEV F3 - Decir que no relacionado con el ejercicio físico; ${ }^{*} \mathbf{p}<0,05 ;{ }^{* *} \mathbf{p}<0,01$. 
$p=0,026)$ y a los 6 meses $\left(t_{(47)}=-1,72, p=0,046\right)$ y en el F1 a los 3 meses $\left(t_{(47)}=-1,92, p=0,030\right)$. Con respecto a la estabilidad temporal, el GC no presentó ninguna puntuación significativa a lo largo del seguimiento.

\section{Discusión}

Debido la implicación de la autoeficacia en los hábitos alimentarios de pacientes con enfermedades crónicas, realizamos un estudio preliminar de las propiedades psicométricas de la escala AEHA en pacientes con SM. La solución factorial presenta dos factores ("Situaciones sociales o estímulo externo para disfrutar de la comida" y "Sentimientos negati-

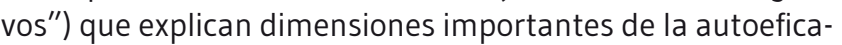
cia. Ya que cuanto menor la autoeficacia en la resistencia a las emociones negativas, mayor la ingesta alimentaria ${ }^{33}$. En otros estudios de validación de instrumentos que evalúan la autoeficacia, se han encontrado factores como "Autoeficacia para conductas alimentarias y evitación de alimento", "Autoeficacia para la evitación de alimentos y actividad física programada" y "Autoeficacia para la actividad física cotidiana y fuerza de voluntad"15, pero básicamente los instrumentos suelen estar agrupados por el tipo de alimento ${ }^{16} \mathrm{o}$ mezclados con actividad física ${ }^{14}$. En la única validación de la AEHA se ha encontrado 3 factores que se refieren a "Situaciones sociales tentadoras", "Sentimientos y estados físicos de humor que pueden despertar una recaída en relación al mantenimiento de la dieta" y "Situaciones tentadoras"17, siendo semejante a la solución encontrada en el presente estudio.

En cuanto a la consistencia interna, los resultados indican que el instrumento es adecuado para medir la autoeficacia relacionada con los hábitos alimentarios. El método de consistencia interna basado en el alfa de Cronbach permite estimar la fiabilidad de un instrumento de medida a través de un conjunto de ítems que se espera que midan el mismo constructo o dimensión teórica. La validez de un instrumento se refiere al grado en que el instrumento mide aquello que pretende medir, a su vez la fiabilidad de la consistencia interna del instrumento se puede estimar con el alfa de Cronbach. La medida de la fiabilidad mediante el alfa de Cronbach asume que los ítems (medidos en escala tipo Likert) miden un mismo constructo y que están altamente correlacionados $^{34}$, por ello cuanto más cerca se encuentre el valor del alfa a 1 mayor es la consistencia interna de los ítems analizados. El método de confiabilidad de las dos mitades se obtiene a partir de una única aplicación del instrumento, ya que al dividirla en mitades equivalentes se obtienen dos puntuaciones de cada persona. La fiabilidad test-retest consiste en aplicar el mismo instrumento por segunda ocasión. En este caso, el coeficiente de confiabilidad es simplemente la correlación entre los resultados de las mismas personas en las dos aplicaciones de la prueba ${ }^{35}$.

Conjuntamente, se observaron asociaciones positivas entre la autoeficacia y la percepción de la capacidad para mantener una alimentación saludable, rechazar alimentos no saludables y la adherencia a la Dieta Mediterránea; y asociaciones negativas con la falta de asertividad general y específica al estilo de vida, con un mayor índice de expresión de la ira y del estrés percibido. Los hallazgos de otros estudios apoyan la relación que encontramos entre la autoeficacia y los factores psicológicos. Específicamente, niveles bajos de estrés percibido y alta autoeficacia dietética se asociaron con menor consumo de grasa y sodio ${ }^{36}$. Igualmente, niveles más altos de apoyo interpersonal y niveles más bajos de estrés percibido se asociaron significativamente con comportamientos saludables ${ }^{37}$. Además, se observó que la baja autoeficacia es uno de los principales factores psicológicos que explican el aumento del estrés en hipertensos ${ }^{38}$. Con respecto a la ira, nuestros resultados están en consonancia con investigaciones que observaron asociaciones de ira con autoculpabilidad y de autoeficacia para manejo de la diabetes con el autocuidado de la misma ${ }^{39}$. Por ello, los profesionales sanitarios deben considerar la relevancia clínica de los factores psicológicos implicados en la alimentación saludable. Además, con respecto a los factores que influyen en el cambio de comportamiento, se puede utilizar factores (análisis de creencias sobre la salud, comportamiento pasado, intención, influencias sociales, control percibido y el contexto del comportamiento) para entender por qué determinados pacientes tienen dificultad para cambiar y también para determinar lo que puede ayudarles ${ }^{40}$.

En relación a la sensibilidad al cambio clínico, encontramos diferencias significativas en el GE (puntuación total a los 3 y 6 meses y en el F1 a los 3 meses), con puntuaciones más elevadas que en la línea base, indicando mayor autoeficacia en el grupo que recibió la intervención. Aun así, es importante destacar que ambos los grupos se encontraban en un rango de puntuación moderado, que se mantuvo en el seguimiento, lo que puede explicar las pocas diferencias encontradas. No obstante, en cuanto a la estabilidad temporal, no encontramos ninguna diferencia en el GC. De modo que la intervención incrementa y/o mantiene la autoeficacia a lo largo del seguimiento. Otros estudios señalan que las mejoras en la autoeficacia y la comprensión del proceso de cambio de comportamiento se asociaron con las actividades de automonitoreo y con el cambio dietético exitoso, a lo 
largo de 4 y 12 meses de seguimiento ${ }^{41}$. La autoeficacia para la dieta también se asoció con el apoyo emocional ${ }^{42}$. Además, la autoeficacia se asoció con el aumento de la ingesta de frutas, vegetales, la disminución de la ingesta de grasa, sodio, el aumento de la actividad física y mayores puntuaciones en la salud cardiovascular en la línea de base ${ }^{43}$. Por ello, la autoeficacia y la planificación pueden desempeñar papeles importantes en los mecanismos que facilitan la fuerza del hábito de estos comportamientos; por lo tanto, deben ser promovidos activamente ${ }^{44}$.

Por otro lado, no se observó ningún efecto del tratamiento en la autoeficacia en pacientes con riesgo cardiovascular ${ }^{45}$, ni para la reducción del peso ${ }^{46}$. Considerando las pocas diferencias significativas encontradas en la autoeficacia, señalamos la posible existencia de otras variables que se relacionan con la autoeficacia en los hábitos alimentarios y la necesidad de más estudios para comprender mejor los mecanismos que subyacen a la autoeficacia.

Como limitaciones, destacamos que la muestra fue exclusivamente clínica lo que evita la generalización de los resultados, así como la implicación de otras variables psicosociales que pueden interferir en los resultados. Además, reportamos datos exploratorios y preliminares y sugerimos que futuros estudios busquen aportar una validación de la AEAH tanto en distintas poblaciones clínicas, como en la población general, así como, intervenciones que consideren el papel de la autoeficacia en el cambio de estilo de vida.

\section{CONCLUSIONES}

Los profesionales sanitarios deben considerar la relevancia clínica de los factores psicológicos implicados en la alimentación saludable. La sensibilidad al cambio clínico indica mayor autoeficacia en el grupo que recibió la intervención. La versión española de la escala AEHA presenta niveles adecuados de validez y fiabilidad en pacientes con síndrome metabólico, demostrando ser útil para evaluar la autoeficacia relacionada a la alimentación.

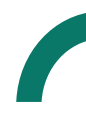

\section{FINANCIACIÓN}

El presente trabajo ha sido financiado por el Ministerio de Educación de Brasil (Fundación Capes) [beca número 556610-0].

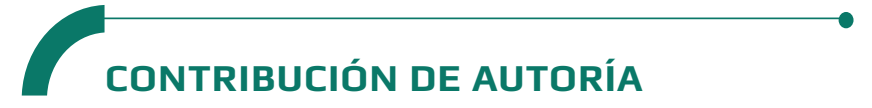

JGS y MIPR han contribuido en la concepción, análisis e interpretación de los datos, así como en la elaboración del artículo.

DSS y GWW han participado en la elaboración del artículo.

NNN y VEC han contribuido en la revisión crítica del contenido y aprobación final del mismo.

Todos/as los/las autores/as han revisado y aceptado la versión final del manuscrito.

\section{CONFLICTO DE INTERESES}

Los autores expresan que no existen conflictos de interés al redactar el manuscrito.

\section{REFERENCIAS}

(1) National Cholesterol Education Program (NCEP) Expert Panel on Detection, Evaluation, and Treatment of High Blood Cholesterol in Adults (Adult Treatment Panel III). Third Report of the National Cholesterol Education Program (NCEP) Expert Panel on Detection, Evaluation, and Treatment of High Blood Cholesterol in Adults (Adult Treatment Panel III) final report. Circulation. 2002; 106(25): 3143-421.

(2) Grundy SM, Brewer HB, Cleeman JI, Smith SC, Lenfant C, National Heart, Lung, and Blood Institute, et al. Definition of metabolic syndrome: report of the National Heart, Lung, and Blood Institute/American Heart Association conference on scientific issues related to definition. Arterioscler Thromb Vasc Biol. 2004; 24(2): e13-18.

(3) Bandura A. Guide for Creating Self-Efficacy Scales. En: Selfefficacy beliefs of adolescents. Greenwich, CT: IAP - Information Age Pub.; 2006. p. 307-37.

(4) Delgado JRP. Estimación psicométrica de la escala de autoeficacia ante conductas de riesgo para adolescentes en México. Psychosoc Interv. 2015; 24(1): 1-7.

(5) Bandura A. Self-efficacy: the exercise of control. Nueva York: W.H. Freeman \& Co; 1997.

(6) Anderson ES, Winett RA, Wojcik JR. Self-regulation, selfefficacy, outcome expectations, and social support: social cognitive theory and nutrition behavior. Ann Behav Med. 2007; 34(3): 304-12.

(7) Chair SY, Wong KB, Tang JY-M, Wang Q, Cheng HY. Social support as a predictor of diet and exercise self-efficacy in patients with coronary artery disease. Contemp Nurse. 2015; 51(2-3): 188-99. 
(8) Lo SWS, Chair SY, Lee FK. Factors associated with healthpromoting behavior of people with or at high risk of metabolic syndrome: Based on the health belief model. Appl Nurs Res. 2015; 28(2): 197-201.

(9) Gardiner CK, Bryan AD. Monetary Incentive Interventions Can Enhance Psychological Factors Related to Fruit and Vegetable Consumption. Ann Behav Med. 2017; 51(4): 599-609.

(10) Luszczynska A, Tryburcy M, Schwarzer R. Improving fruit and vegetable consumption: a self-efficacy intervention compared with a combined self-efficacy and planning intervention. Health Educ Res. 2007; 22(5): 630-8.

(11) Göhner W, Schlatterer M, Seelig H, Frey I, Berg A, Fuchs R. Two-year follow-up of an interdisciplinary cognitive-behavioral intervention program for obese adults. J Psychol. 2012; 146(4): 371-91.

(12) Karl JP, Cheatham RA, Das SK, Hyatt RR, Gilhooly CH, Pittas $A G$, et al. Effect of glycemic load on eating behavior selfefficacy during weight loss. Appetite. 2014; 80: 204-11.

(13) Guertin C, Rocchi M, Pelletier LG, Émond C, Lalande G. The role of motivation and the regulation of eating on the physical and psychological health of patients with cardiovascular disease. ] Health Psychol. 2015; 20(5): 543-55.

(14) Románi Y, Díaz B, Cárdenas M, Zoraide L. Construcción y validación del Inventario Autoeficacia Percibida para el Control de Peso. Clin Salud. 2007; 18(1): 45-56.

(15) Guzmán Saldaña RME, Gómez Peresmitré G. Análisis discriminante del Inventario de Autoeficacia para Control de Peso en adolescentes mexicanos. Psicol Salud. 2011; 21(2): 157-64.

(16) Palacios J, Ramírez V, Anaya M, Hernández HL, Martínez R. Evaluación psicométrica de una escala de autoeficacia de la conducta alimentaria. Rev Chil Nutr. 2017; 44(1): 95-102.

(17) Boff R de M. Evidências psicométricas das escalas de autoeficácia para regular hábito alimentar e auto-eficácia para regular exercício físico. [Porto Alegre, Brasil]: Pontifícia Universidade Católica do Rio Grande do Sul; 2012. Disponible en: http://tede2.pucrs.br:80/tede2/handle/tede/832

(18) Garcia-Silva J, Peralta-Ramírez MI, Navarrete Navarrete N, Silva-Silva D, Caballo VE. Validez y fiabilidad de la escala de autoeficacia para el ejercicio físico en pacientes con síndrome metabólico. Rev Esp Salud Publica. 2018; 92: e201808046.

(19) Folstein MF, Folstein SE, McHugh PR, Fanjiang G. MMSE: examen cognoscitivo mini-mental: manual. Madrid: TEA; 2002.

(20) Becoña E, López A, Fernández del Río E, Míguez MC, Castro J. Spanish adaptation of the NDSS (Nicotine Dependence Syndrome Scale) and assessment of nicotine-dependent individuals at primary care health centers in Spain. Span ] Psychol. 2010; 13(2): 951-60.

(21) Downer MK, Gea A, Stampfer M, Sánchez-Tainta A, Corella $D$, Salas-Salvadó J, et al. Predictors of short- and long-term adherence with a Mediterranean-type diet intervention: the PREDIMED randomized trial. Int J Behav Nutr Phys Act. 2016; 13: 67.

(22) Spielberger CD. STAXI-2: inventario de expresión de ira estadorasgo: manual. Madrid: TEA Ediciones; 2001.

(23) Gambrill ED, Richey CA. An assertion inventory for use in assessment and research. Behav Ther. 1975; 6(4): 550-61.

(24) Carrasco I, Clemente M, Llavona L. Analysis of Gambrills and Richey's Assertion Inventory. Estudios de Psicología. 1989; 10(37): 63-74.
(25) Salazar IC, Roldán GM, Garrido L, Ramos JM. La asertividad y su relación con problemas emocionales y el desgaste en profesionales sanitarios. Behav Psychol. 2014; 22(3): 523-49.

(26) Caballo VE, Salazar IC, Irurtia MJ, Olivares J, Toledo R. Evaluación de las habilidades sociales: estructura factorial y otras propiedades psicométricas de cuatro medidas de autoinforme. Behav Psychol. 2014; 22(3): 375-99.

(27) Remor E. Psychometric properties of a European Spanish version of the Perceived Stress Scale (PSS). Span J Psychol. 2006; 9(1): 86-93.

(28) García J, Caballo VE, Peralta Ml, Lucena-Santos P, Navarrete $N$. Cuestionario de asertividad centrado en el estilo de vida (CACEV) en pacientes con síndrome metabólico: desarrollo y validación. Behav Psychol. 2017; 25(2): 349-69.

(29) Agencia Española de Consumo, Seguridad Alimentaria y Nutrición (AECOSAN). Estrategia NAOS: Estrategia para la Nutrición, Actividad Física y Prevención de la Obesidad [Internet]. AECOSAN. 2005 [citado 25 de noviembre de 2012]. Disponible en: http://www.aecosan.msssi.gob.es/AECOSAN/ web/nutricion/seccion/estrategia_naos.htm

(30) de Winter JCF, Dodou D, Wieringa PA. Exploratory Factor Analysis With Small Sample Sizes. Multivariate Behav Res. 2009; 44(2): 147-81.

(31) MacCallum RC, Widaman KF, Zhang S, Hong S. Sample size in factor analysis. Psychol Methods. 1999; 4(1): 84-99.

(32) Field A. Discovering Statistics Using IBM SPSS Statistics. 4th ed. Londres: Sage Publications Ltd.; 2013.

(33) Shimpo M, Fukkoshi Y, Akamatsu R. Correlations between selfefficacy in resisting six temptations and dietary energy and macronutrient intake at each meal. Eat Behav. 2014; 15(4): 563-6.

(34) Welch S, Comer J. Quantitative methods for public administration: techniques and applications. 2a ed. Boston, MA: Brooks/Cole Pub. Co.; 1988.

(35) Cappello HM, Aguirre MI, Castro RD, Cervantes RD, Infante JI, Marín R. Estudio del análisis de la consistencia interna y la validez de construcción de una prueba en el programa de enseñanza vivencial de las ciencias. SOCIOTAM. 2004; 14(2): 47-72.

(36) Nastaskin RS, Fiocco AJ. A survey of diet self-efficacy and food intake in students with high and low perceived stress. Nutr ]. 2015; 14: 42.

(37) Cho JH, Jae SY, Choo ILH, Choo J. Health-promoting behaviour among women with abdominal obesity: a conceptual link to social support and perceived stress. J Adv Nurs. 2014; 70(6): 1381-90.

(38) Trovato GM, Pace P, Martines GF, Trovato FM, Pirri C, Catalano D. Stress, abdominal obesity and intrarenal resistive index in essential hypertension. Clin Ter. 2012; 163(4): 299-305.

(39) DePalma MT, Trahan LH, Eliza JM, Wagner AE. The Relationship between Diabetes Self-efficacy and Diabetes Self-care in American Indians and Alaska Natives. Am Indian Alsk Native Ment Health Res. 2015; 22(2): 1-22.

(40) Barley E, Lawson V. Using health psychology to help patients: theories of behaviour change. Br J Nurs. 2016; 25(16): 924-7.

(41) Gillison F, Stathi A, Reddy P, Perry R, Taylor G, Bennett P, et al. Processes of behavior change and weight loss in a theorybased weight loss intervention program: a test of the process model for lifestyle behavior change. Int ] Behav Nutr Phys Act. 2015; 12: 2 
(42) Chair SY, Wong KB, Tang JY-M, Wang Q, Cheng HY. Social support as a predictor of diet and exercise self-efficacy in patients with coronary artery disease. Contemp Nurse. 2015; 51(2-3): 188-99.

(43) Brewer LC, Balls-Berry JE, Dean P, Lackore K, Jenkins S, Hayes SN. Fostering African-American Improvement in Total Health (FAITH!): An Application of the American Heart Association's Life's Simple $7^{\text {TM }}$ among Midwestern African-Americans. ] Racial Ethn Health Disparities. 2017; 4(2): 269-81.

(44) Storm V, Dörenkämper J, Reinwand DA, Wienert J, De Vries $\mathrm{H}$, Lippke S. Effectiveness of a Web-Based Computer-Tailored Multiple-Lifestyle Intervention for People Interested in
Reducing their Cardiovascular Risk: A Randomized Controlled Trial. J Med Internet Res. 2016; 18(4): e78.

(45) Arambepola C, Ricci-Cabello I, Manikavasagam P, Roberts N, French DP, Farmer A. The Impact of Automated Brief Messages Promoting Lifestyle Changes Delivered Via Mobile Devices to People with Type 2 Diabetes: A Systematic Literature Review and Meta-Analysis of Controlled Trials. ] Med Internet Res. 2016; 18(4): e86.

(46) Schiel R, Kaps A, Stein G, Steveling A. Predictors and determinants for weight reduction in overweight and obese children and adolescents. Z Evid Fortbild Qual Gesundhwes. 2016; 118-119: 31-9. 